\title{
A modified tilted-pulse-front excitation scheme for efficient terahertz generation in $\mathrm{LiNbO}_{3}$
}

\author{
$\underline{\text { S.B. Bodrov, }}^{1,2}$ A.N. Stepanov ${ }^{2}$, E.A. Burova ${ }^{1}$, and M.I. Bakunov ${ }^{1}$ \\ ${ }^{1}$ University of Nizhny Novgorod, Nizhny Novgorod 603950 Russia \\ ${ }^{2}$ Institute of Applied Physics of the Russian Academy of Sciences, Nizhny Novgorod 603950 Russia
}

Pumping of $\mathrm{LiNbO}_{3}$ by femtosecond optical pulses with tilted intensity front (pulse front) allows one to obtain high efficiency of optical-to-terahertz conversion (up to $1 \%$ at room temperature [1]), large energy of generated terahertz pulses (up to sub-mJ level [2]), and high strength of terahertz fields (exceeding 1 $\mathrm{MV} / \mathrm{cm}$ [3]). Strong terahertz pulses are in demand for various applications, including terahertz-driven particle acceleration [4], terahertz nonlinear spectroscopy [5], and terahertz streaking techniques [6].

Tilted-pulse-front optical pulses are conventionally obtained by diffraction of a laser pulse off an optical grating. A lens (or two-lens telescope) is used to relay-image such pulses into the $\mathrm{LiNbO}_{3}$ crystal $[7,8]$. In order to maximize the optical-to-terahertz conversion efficiency, the tilt angle $\alpha$ in the crystal should be close to a value defined by the equation $\cos \alpha=n_{g} / n_{\mathrm{THz}}$, where $n_{\mathrm{g}}$ is the optical group refractive index and $n_{\mathrm{THz}}$ is the terahertz phase refractive index. Additionally, it is postulated that a good quality of the terahertz beam can be achieved if the image of the grating is parallel both to the pulse front and to the exit surface of the crystal $[7,8]$.

Here, we extend the analysis of the tilted-pulsefront excitation scheme $[7,8]$ to a more general geometry, namely, with the pulse front not parallel to the exit surface of the crystal (the grating image is still assumed to be parallel to the surface).

In our theory, the generated terahertz radiation is found analytically from the Maxwell equations with a nonlinear polarization included as a source $[9,10]$. The nonlinear polarization is found as a result of optical rectification of the tilted-pulse-front optical pulse, which experiences effects of both material and angular dispersion.

At first, we consider the conventional geometry of the tilted-pulse-front excitation scheme, i.e., with the pulse front parallel to the exit surface of the crystal, but for an arbitrary orientation of the grating image plane, defined by the angle $\theta$ [Fig. 1(a)]. Figures 1 (b)-(e) show the effects of the grating image orientation on the efficiency and spatial distribution of the generated spectrum along the exit surface of the crystal ( $y$-axis). In the optimal case, when $\theta=\alpha$, the spectrum distribution is rather uniform [Fig. 1(d)]. For $\theta \neq \alpha$, the bandwidth and shape of the spectrum varies considerably with $y$ [Figs. 1(c) and 1(e)]. The spectral non-uniformity across the terahertz beam can give strong divergence of the beam and prevent its further focusing. Interestingly, for $\theta<\alpha$, the spectral amplitude in the interval $-5<y<-1 \mathrm{~mm}$ is larger than for the optimal configuration $\theta \neq \alpha$ [Figs. 1(c) and 1(d)]. As a result, the energy of generated terahertz radiation does not decrease and even somewhat increases at a deviation of $\theta$ from the optimal value $\theta=\alpha$ to $\theta<\alpha$ [Fig. 1(b)]. In the case $\theta>\alpha$, the terahertz energy [Fig. 1(b)] and maximal spectral amplitude [Fig. 1(e)] decrease rapidly with deviation of $\theta$ from $\alpha$.
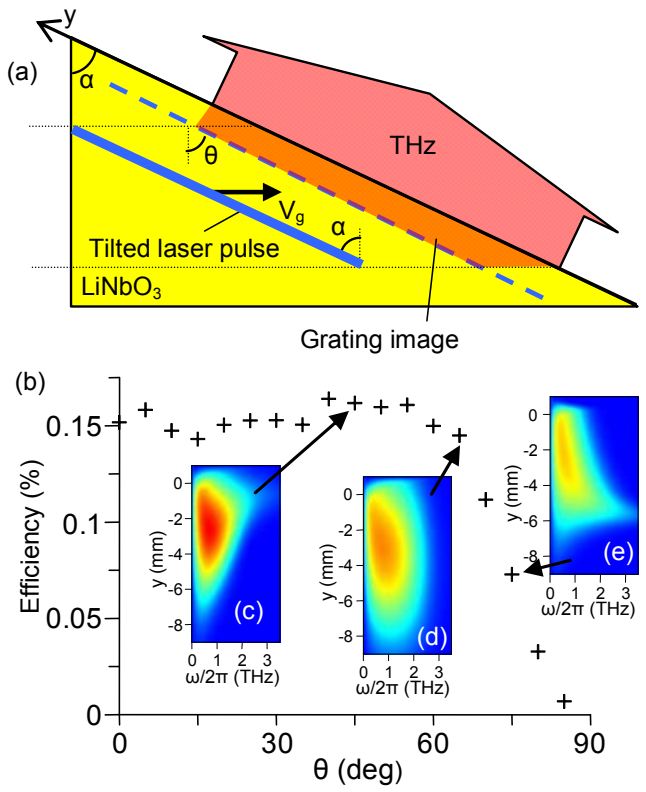

Fig. 1. (a) The conventional geometry of the tilted-pulsefront excitation technique with the pulse front parallel to the exit surface of the crystal. (b) Efficiency as a function of $\theta$. (c-e) Distribution of the amplitude spectrum near the exit surface for (c) $\theta=45^{\circ}$, (d) $\theta=\alpha=63.3^{\circ}$, and (e) $\theta=75^{\circ}$. The optical pulse has a $3 \mathrm{~mm}$ transverse size and 100 fs duration.

Now let us consider a more general geometry, depicted in Fig. 2, with the pulse front not parallel to the exit surface of the crystal (the grating image plane is assumed to be parallel to the exit surface). In this geometry, the generated terahertz radiation propagates normally to the pump pulse front and, therefore, impinges obliquely on the exit surface of the crystal. To prevent total internal reflection of the radiation and minimize Fresnel losses, a Si prism should be attached to the exit surface of the $\mathrm{LiNbO}_{3}$ crystal (Fig. 2). 


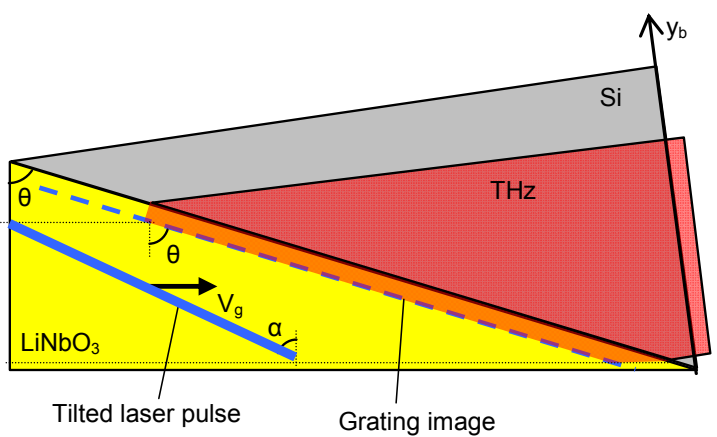

Fig. 2. The generalized tilted-pulse-front excitation geometry with the pulse front not parallel to the exit surface of the crystal.

Figures 3(a) and 3(b) show the distributions of the amplitude frequency spectrum along the exit boundary of the Si prism ( $y_{b}$-axis) for two different angles $\theta=45^{\circ}$ and $\theta=75^{\circ}$, respectively. The distributions are rather uniform, similar to the optimal case in Fig. 1(d), and have practically the same spectral bandwidth for both angles. However, the maximal value of the spectral amplitude and size of its spatial localization differ substantially. The maximal value of the spectral density is two times higher for $\theta=45^{\circ}$ than for $\theta=75^{\circ}$, but the size of the terahertz beam is $2-3$ times lager for $\theta=75^{\circ}$. The last fact is explained by a larger projection of the pump beam on the exit boundary of the $\mathrm{LiNbO}_{3}$ crystal.
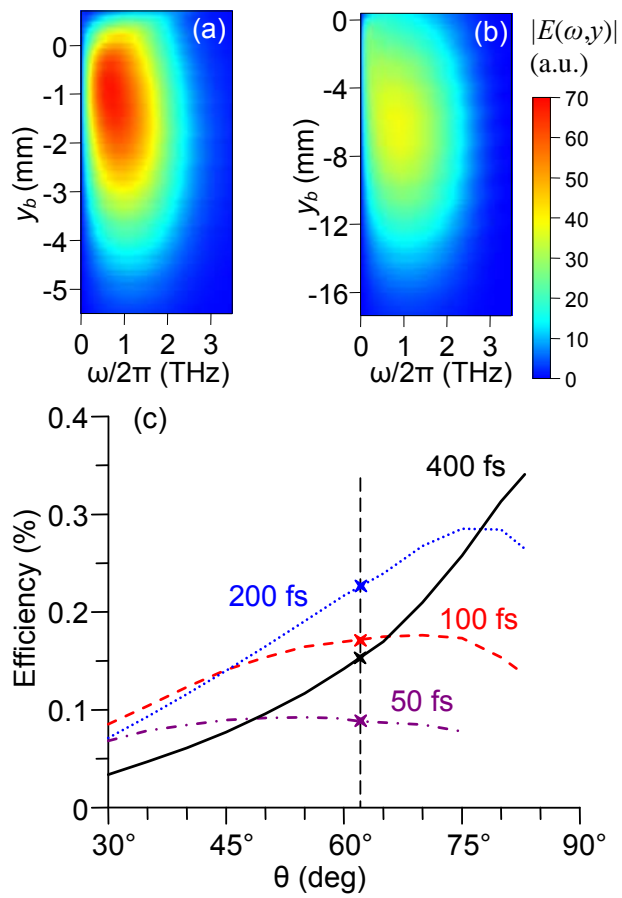

Fig. 3. Distribution of the amplitude spectrum along the exit boundary of the crystal for (a) $\theta=45^{\circ}$ and (b) $\theta=75^{\circ}$. (c) The conversion efficiency as a function of $\theta$ for different pulse durations. The optical pulse energy is fixed and corresponds to $10 \mathrm{GW} / \mathrm{cm}^{2}$ at a pulse duration of $100 \mathrm{fs}$. The pump beam transverse size is $3 \mathrm{~mm}$. Vertical line marks the optimal case $\theta=\alpha$.

Figure 3(c) shows the optical-to-terahertz conversion efficiency as a function of $\theta$ for different pump pulse durations. For a short pump pulse (50 fs), the efficiency is almost independent of $\theta$ in the interval from $30^{\circ}$ to $75^{\circ}$ and decreases rapidly for $\theta>75^{\circ}$. With increase of the pump pulse duration, the maximum becomes more pronounced and shifts to larger $\theta$. The maximal efficiency also increases (for a fixed energy of the pump pulse) and for pulse duration $>100$ fs exceeds the efficiency in the optimal case. For the pump pulse of a 400 fs duration, the maximum efficiency at $\theta \approx 85^{\circ}$ is about two times higher than in the optimal geometry, i.e., at $\theta=\alpha=63.3^{\circ}$.

To conclude, we have shown that a deviation of the grating image plane from the optimal orientation in the tilted-pulse-front excitation technique leads to a deterioration of the terahertz beam quality but does not affect significantly the terahertz energy (in the case $\theta<\alpha)$. We have proposed a modified variant of the tilted-pulse-front excitation scheme with the pulse front not parallel to the exit surface of the crystal. We have shown that this geometry allows one to double the terahertz generation efficiency for long ( $>200 \mathrm{fs}$ ) laser pulses still preserving a high quality of the generated terahertz beam.

This work was supported by the Russian Science Foundation (RSF) (18-19-00486).

\section{References}

1. S.-W. Huang, E. Granados, W. R. Huang, K.-H. Hong, L. E. Zapata, and F. X. Kärtner. High conversion efficiency, high energy terahertz pulses by optical rectification in cryogenically cooled lithium niobate // Opt. Lett. 2013. V. 38. P. 796.

2. J. A. Fülöp, Z. Ollmann, C. Lombosi, C. Skrobol, S. Klingebiel, L. Pálfalvi, F. Krausz, S. Karsch, and J. Hebling. Efficient generation of $\mathrm{THz}$ pulses with $0.4 \mathrm{~mJ}$ energy // Opt. Express. 2014. V. 22. P. 20155.

3. H. Hirori, A. Doi, F. Blanchard, and K. Tanaka. Single-cycle terahertz pulses with amplitudes exceeding $1 \mathrm{MV} / \mathrm{cm}$ generated by optical rectification in $\mathrm{LiNbO}_{3}$ // Appl. Phys. Lett. 2011. V. 98. 091106.

4. E. A. Nanni, W. R. Huang, K.-H. Hong, K. Ravi, A. Fallahi, G. Moriena, R. J. Miller, and F. X. Kärtner. Terahertz-driven linear electron acceleration // Nat. Commun. 2015. V. 6. P. 8486.

5. S. Bodrov, Yu. Sergeev, A. Murzanev, and A. Stepanov Terahertz induced optical birefringence in polar and nonpolar liquids // J. Chem. Phys. 2017. V. 147. P. 084507.

6. U. Fruhling, Light-field streaking for FELs, // J. Phys. B. 2011. V. 44. P. 243001.

7. J. A. Fülöp, L. Pálfalvi, G. Almási, and J. Hebling. Design of high-energy terahertz sources based on optical rectification // Opt. Express. 2010. V. 18. P. 12311.

8. L. Tokodi, J. Hebling, and L. Pálfalvi. Optimization of the tilted-pulse-front terahertz excitation setup containing telescope // J. Infrared Milli. Terahz. Waves. 2017. V. 38. P. 22.

9. M. I. Bakunov, S. B. Bodrov, and E. A. Mashkovich. Terahertz generation with tilted-front laser pulses: dynamic theory for low-absorbing crystals // J. Opt. Soc. Am. B 2011. V. 28. P. 1724.

10. M. I. Bakunov and S. B. Bodrov. Terahertz generation with tilted-front laser pulses in a contact-grating scheme // J. Opt. Soc. Am. 2014. V. 31. P. 2549. 